\title{
Floor Field Model Based on Cellular Automata for Simulating Indoor Pedestrian Evacuation
}

\author{
Xiao Yang, Binxu Wang, and Zheng Qin \\ School of Software, Tsinghua University, Beijing 100084, China \\ Correspondence should be addressed to Xiao Yang; yangxiao356@sohu.com
}

Received 6 April 2015; Accepted 15 July 2015

Academic Editor: Xiaoyu Song

Copyright ( 2015 Xiao Yang et al. This is an open access article distributed under the Creative Commons Attribution License, which permits unrestricted use, distribution, and reproduction in any medium, provided the original work is properly cited.

\begin{abstract}
A new static floor field method for simulations of evacuation processes based on cellular automaton was presented in this paper. This model applies an inertia static floor field approach to describe the interaction between the pedestrians and the cell. Here we study a rather simple situation and a complex scenario. We simulate and reproduce Seyfried's field experiments at the Research Centre Jülich and use its empirical data to validate our model. The concept of scenario-familiarity of the crowd has been proposed to explain the model. It is shown that the variation of the model parameters deeply impacts the evacuation efficiency. The relation between minimal evacuation times and the knowledge of the exit that the pedestrian acknowledges is discussed.
\end{abstract}

\section{Introduction}

With the development of economy and the speeding up of urbanization, the number of urban population has increased dramatically in all countries. The possibility of congestion occurring also gradually increased; in the face of such a large stream of people, some public facilities produce all kinds of evacuation problems, and reasonable evacuation of pedestrian flow will play a positive role for the construction of wisdom city. How to better handle the evacuation problem becomes a hotspot in the research field, also it brings the difficulties. This is especially true in developing countries; the big events in these countries tend to have more people. Now more and more scholars begin to pay close attention to public safety and the research of pedestrian evacuation; the results will be widely used in the emergency evacuation simulation, the safe evacuation design, potential risk prediction, and so on.

\section{Floor Field Model}

Floor field is a kind of widely used cellular automata model; Burstedde et al. [1] proposed static floor field and dynamic floor field in 2001; many scholars improved the different aspects of potential model and got a lot of extension of the model.

A cellular automaton (pl.: cellular automata $(\mathrm{CA})$ ) is a discrete model studied in computability theory, mathematics, physics, complexity science, theoretical biology, and microstructure modeling. Cellular automata are also called cellular spaces, tessellation automata, homogeneous structures, cellular structures, tessellation structures, and iterative arrays [2]; a standard cellular automaton $A$ can be defined as $A=(L, d, S, N, f), L$ is the cellular space, $d$ is the number of dimensions of the cellular space, $S$ is the limited dispersed collection, $N$ is the collection of all the cells in its neighborhood, and $f$ is the local map or rules.

Kirchner et al. [3] presented the irrationality that all the pedestrians share the same speed in evacuation; Ma et al. [4] and Guo and Huang [5] use the smaller cell to simulate the pedestrian evacuation and succeed in showing some selforganizing phenomenon; Yanagisawa et al. [6] apply floor field model to the turning corner; Chraibi et al. [7] proposed the force-based models of pedestrian dynamics; Zeng et al. [8] used a local-view floor field model to optimize the efficiency of the evacuation.

The floor field adjusts the movement probability by static floor field and real-time dynamic floor field to realize 


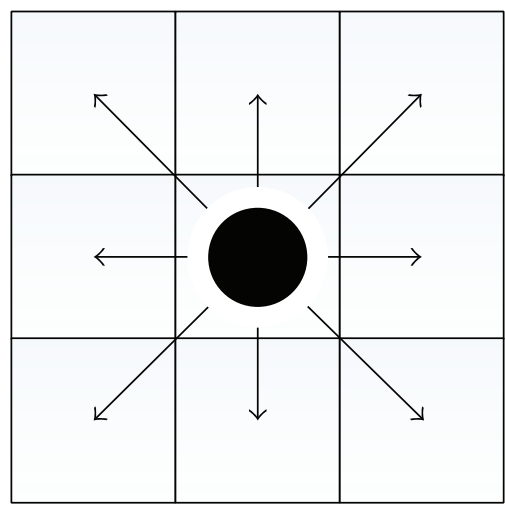

(a)

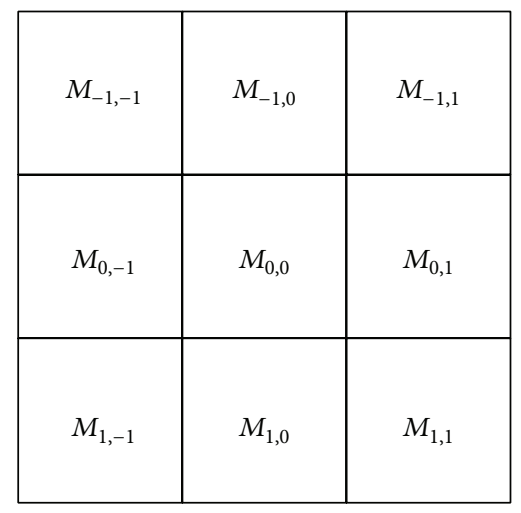

(b)

FIGURE 1: Move selection and preference matrix.
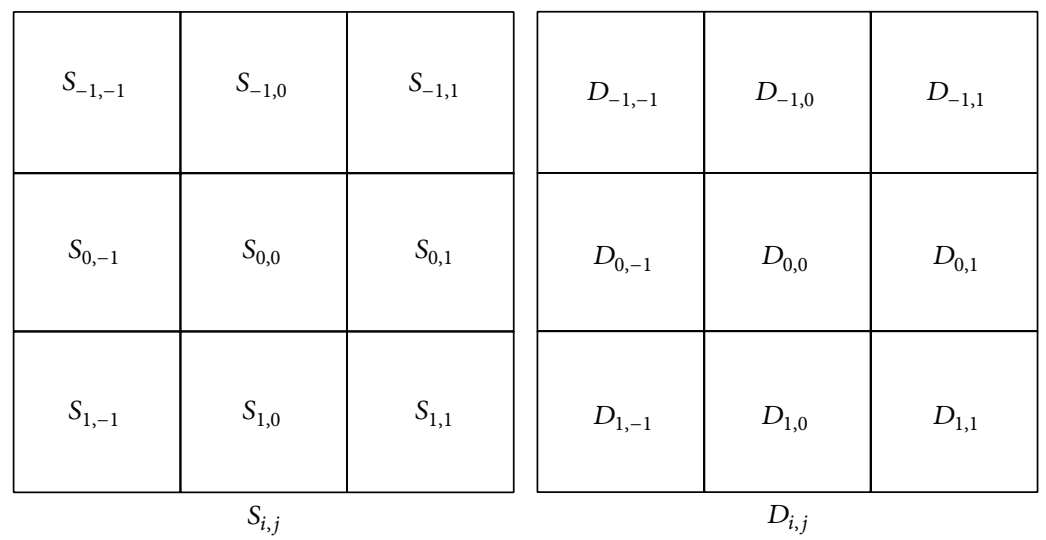

Figure 2: Static and dynamic floor field matrix.

the interaction of the pedestrian. The static floor field is defined as the distance from the cell to the exit (Formula (1)), it is constant after the initialization, and the static floor field value of the cell is in inverse proportion to the distance to the exit; the dynamic floor field is changing as the evacuation goes; the movement information of the pedestrian will be recorded, along with the diffusion and decay. The floor field model gives expression to terrain information of the scene; by using this model, pedestrian follow and automatic canalization can be simulated:

$$
\begin{aligned}
S_{i, j} & =\min _{\left(i_{\tau_{s}}, j_{\tau_{s}}\right)}\left\{\max _{\left(i_{l}, j_{l}\right)}\left\{\sqrt{\left(i_{\tau_{s}}-i_{l}\right)^{2}+\left(j_{\tau_{s}}-j_{l}\right)^{2}}\right\}\right. \\
& \left.-\sqrt{\left(i_{\tau_{s}}-i\right)^{2}+\left(j_{\tau_{s}}-j\right)^{2}}\right\} .
\end{aligned}
$$

The move selection should be predefined (Figure 1(a)), and then set up a corresponding preference matrix (Figure 1(b)), also the static floor field matrix and the dynamic floor field matrix (Figure 2). For the different distance between the vertical direction and the diagonal direction, the moving speed of the two directions can be set to $1: \sqrt{2}$ approximately.
The pedestrian moving probability $p_{i j}$ in Burstedde et al's paper [1] is as follows:

$$
p_{i j}=N M_{i j} D_{i j} S_{i j}\left(1-n_{i j}\right) .
$$

Kirchner and Schadschneider [9] improved the formula:

$$
p_{i j}=N \exp \left(k_{D} D_{i j}\right) \exp \left(k_{S} S_{i j}\right)\left(1-n_{i j}\right) .
$$

The data coming from Formula (3) is closer to the actual situation, so we choose this formula. The description of the formula parameters is shown in Table 1 :

$$
N=\left[\sum_{(i, j)} \exp \left(k_{D} D_{i j}\right) \exp \left(k_{S} S_{i j}\right)\left(1-n_{i j}\right)\right]^{-1} .
$$

\section{The Improved Floor Field Model}

First, we reproduce Seyfried's experiment by the standard floor field model; then we discuss the efficiency of the new floor field model. The static and dynamic floor field calculation methods were improved, respectively; we put forward a new static floor field calculation method and compared with classical calculation method by simulation 


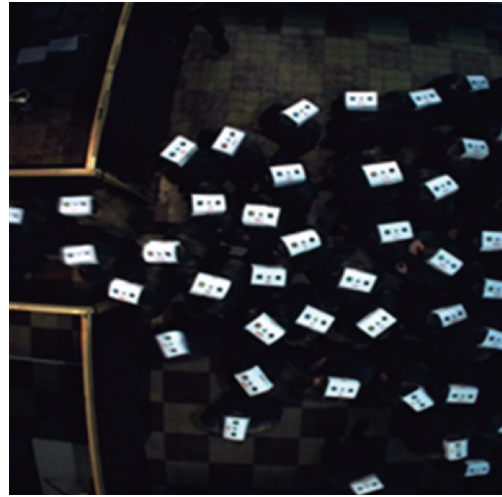

(a)

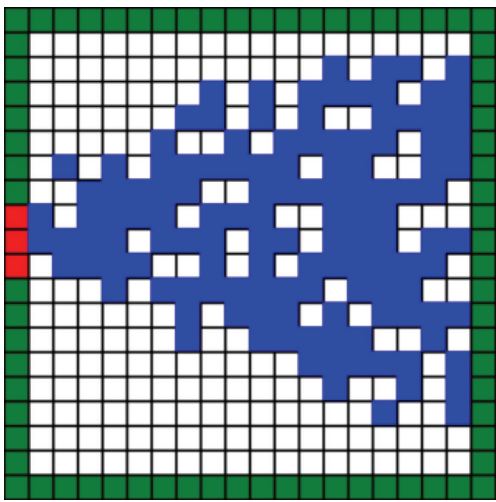

(b)

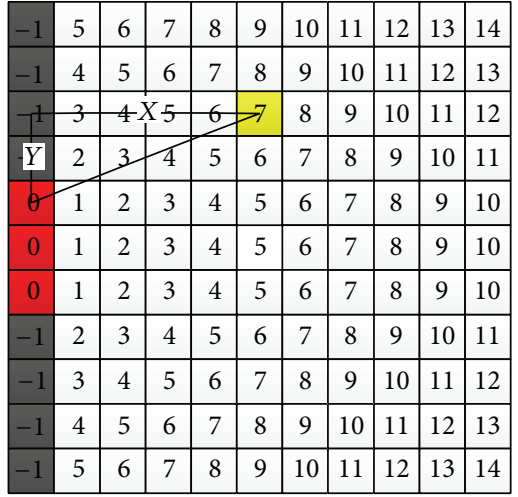

(c)

FIgURE 3: Experiment simulation and the values of static floor field.

TABLE 1: Formula parameter.

\begin{tabular}{ll}
\hline Parameter & Interpretation \\
\hline$M_{i j}$ & Pedestrian moving preference \\
$n_{i j}$ & Cell occupy mark (occupied = 1 and unoccupied = \\
$k_{D} \& k_{S}$ & 0 Static and dynamic floor field weight \\
$D_{i j} \& S_{i j}$ & Static and dynamic floor field value \\
$N$ & Normalization coefficient \\
\hline
\end{tabular}

experiments. We compare the improved FF model with the standard FF model.

\subsection{Model Description}

3.1.1. Seyfried's Experiment and Analysis. Seyfried et al. [10] considered that there are a variety of models proposed to discuss the pedestrian dynamic evacuation, but only few attempts try to validate these approaches; it is necessary to have more reliable data that can be used as basis for validation and calibration which then would allow making quantitative predictions based on computer simulations. The pedestrian evacuation experiment is data-oriented behavior [11]; we still need much more data to test the accuracy of the models.

The experiments were recorded by video cameras and pedestrian trajectories were extracted from the videos; then we reproduced the pedestrian dynamics of the experiment by the simulated model based on floor field model. The simulated result is showed in Figure 3(b). Although Seyfried's experiment has focused on the influence of the bottleneck to the pedestrian, it revealed the evacuation time and the density of the pedestrian evacuation. We set up our model to simulate the section from the beginning to the moment the pedestrian is about to walk into the bottleneck.

3.1.2. The New Static Floor Field Model. The floor field is based on the cellular automata model; the state of the next moment of each cell is dependent on the neighbors. Since this simulated indoor pedestrian evacuation process, setting

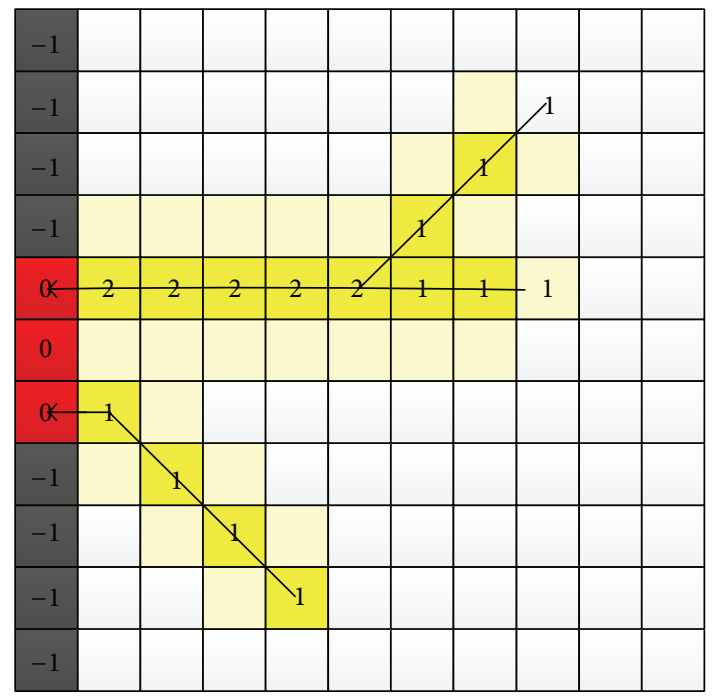

FIGURE 4: Dynamic floor field (light yellow cell stands for the cells diffused by Von Neumann).

up and down the room in addition to the four borders where exports are obstacles, pedestrians cannot pass. Each cell has the properties of occupation and the static floor field. First, determine the direction of the pedestrian, begin with what the surrounding cell can move, mark it as a selected target, and then subtract the value of the static floor field between the pedestrian cell and the selected neighbor cell. If the difference is positive, indicating that there is great willingness for pedestrians moving in that direction, there is a greater probability that the choice of the cell, if the value is negative, indicates that there is little willingness for pedestrians walking in that direction; there is a small probability of selecting the cell point. If the surrounding grid points are not available, it can only wait.

This model is defined in a two-dimensional plane $(50 \times$ 50), as shown in Figure 3(c). Reference [12] stated the space that one pedestrian takes; each person occupies about $0.4 \times 0.4 \mathrm{~m}^{2}$; the simulation is dispersed; the speed of 


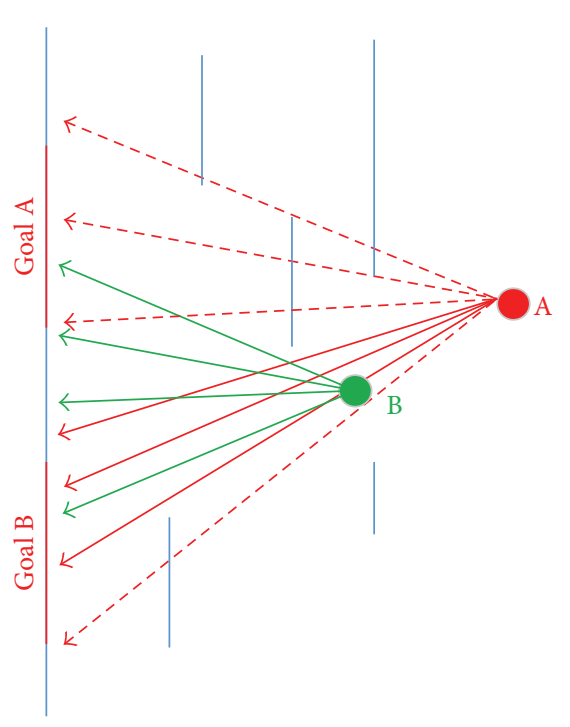

(a)

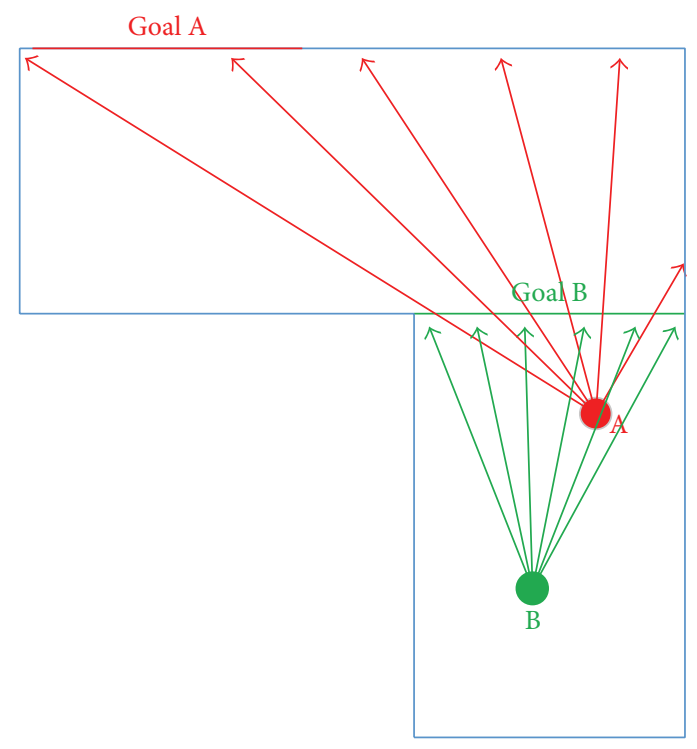

(b)

Figure 5: (a) The visibility of the pedestrian; the destination may change when the pedestrian moved (moving from A to B). (b) Different positions bring about different goals.

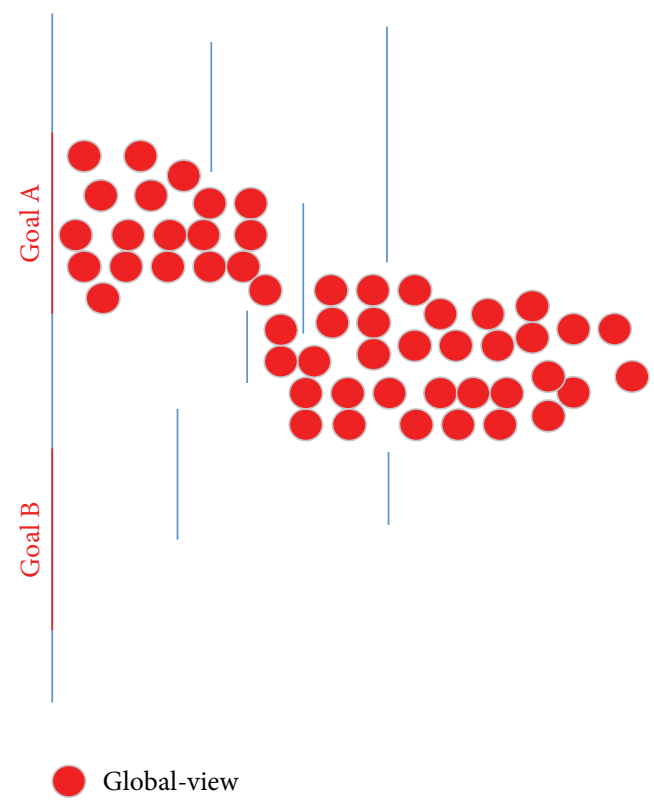

(a)

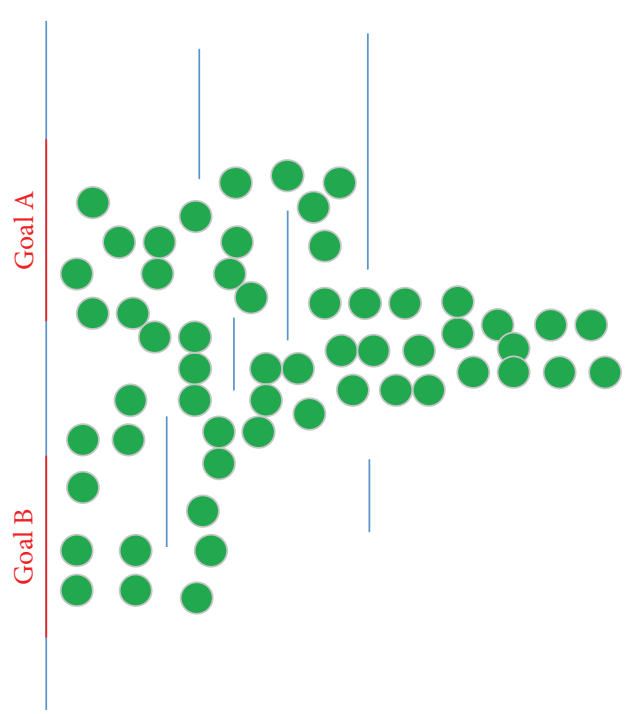

Local-view

FIGURE 6: The sketch of global-view model and local-view model.

the pedestrian is 1 (cell/step); every time step the pedestrian chooses to move towards the direction of the selected step, or in situ waiting. Figure 3(c) showed part of the values of the static floor field. The static floor field $S$ can be simply defined as the Manhattan distance between the cell and the exit; that is, these two line segments on the axis of the projection distance are summed on the rectangular coordinate system; the static floor field of the marked cell in Figure 3(c) is $X+Y$.

In this paper, an improved algorithm of static floor field for some specific scenarios was proposed; for example, the static floor field reflects how familiar the pedestrian is with the export message in the indoor evacuation model, with static floor field weigh coefficient, after the cognizance of the exit message, especially to some pedestrian far from the exit; the pedestrian always chooses the same direction at the first few steps, which may be blocked by the other obstacles, but with the exit of the running direction the case of more than a certain threshold will not change; we call it inertia static floor field; walking along this direction; simply record the value of the static floor field of the cell that has passed, until 


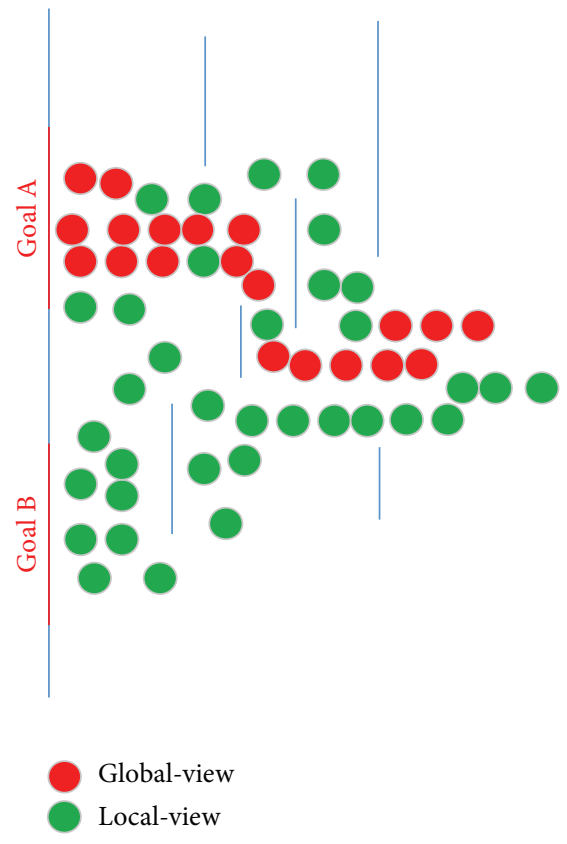

(a)

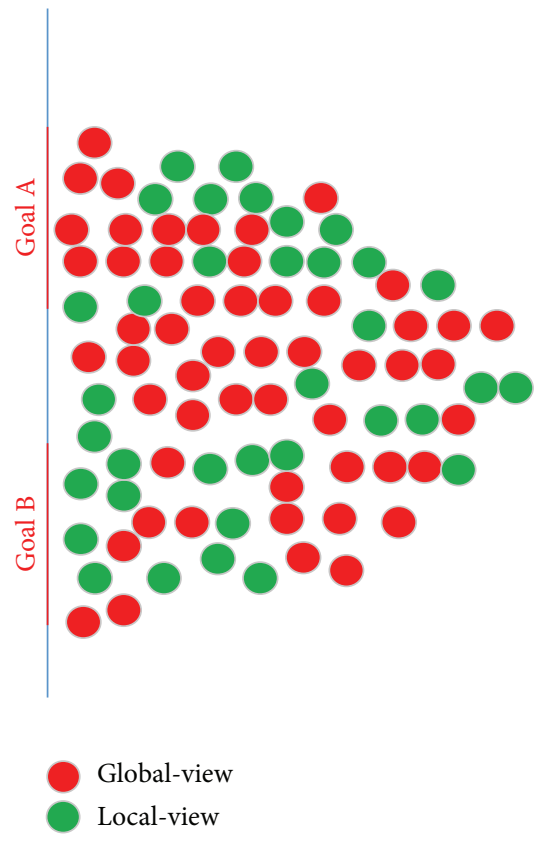

(b)

FIGURE 7: (a) The new model we proposed (b) using our model in the simple scenario.
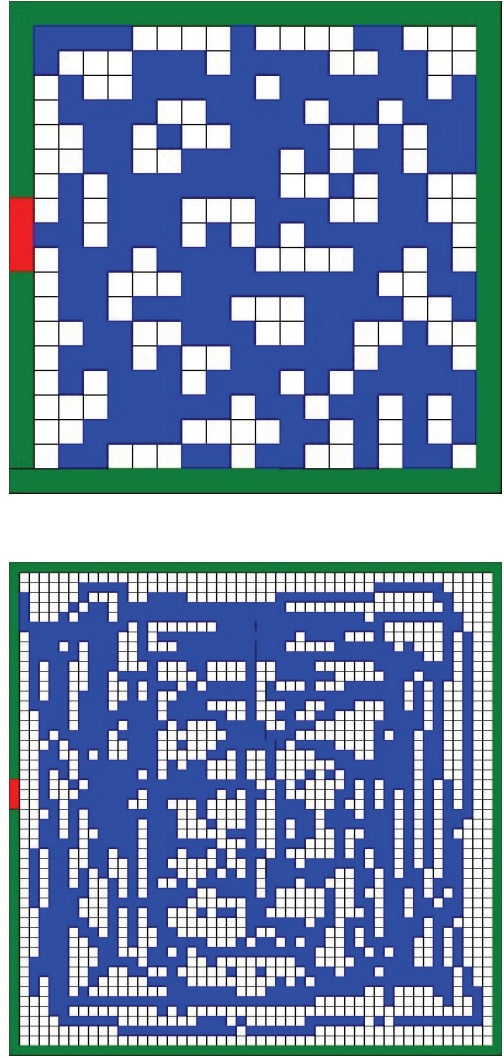

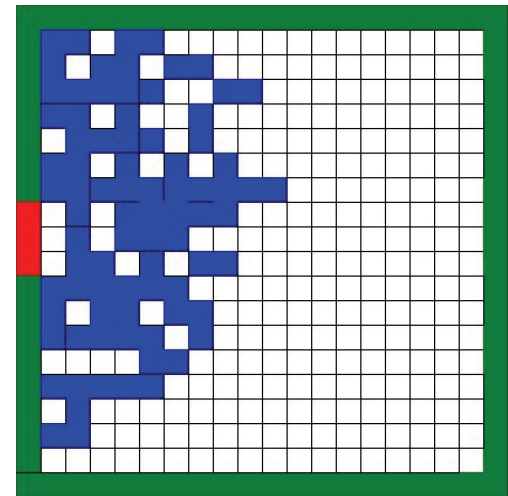

(a) $20 \times 20$ scenario

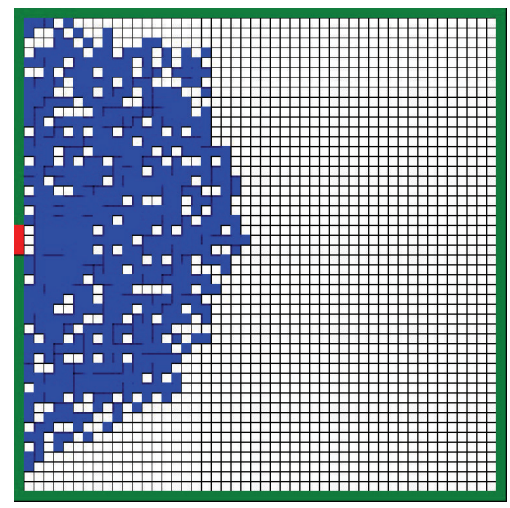

(b) $100 \times 100$ scenario
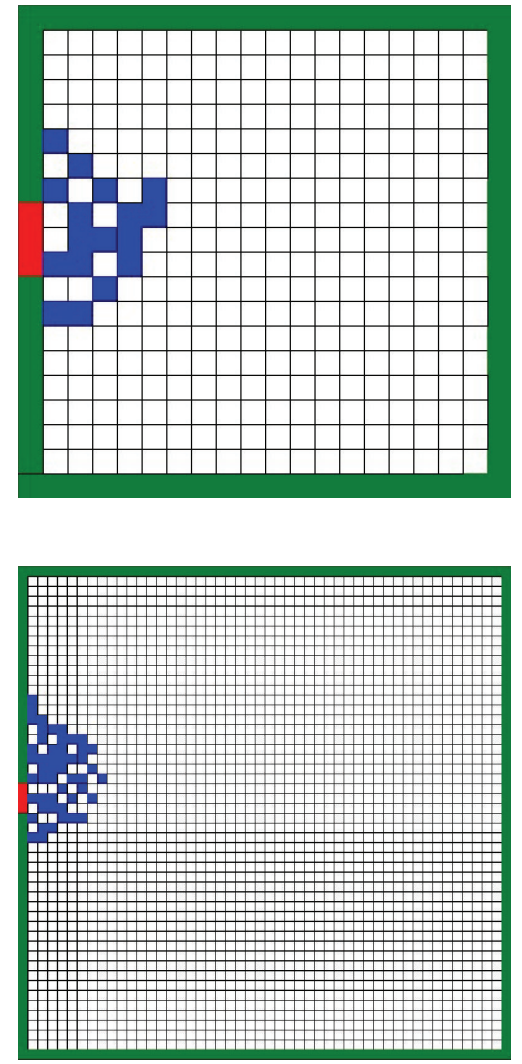

FIgURE 8: Pedestrian evacuation simulation in simple scenario. 

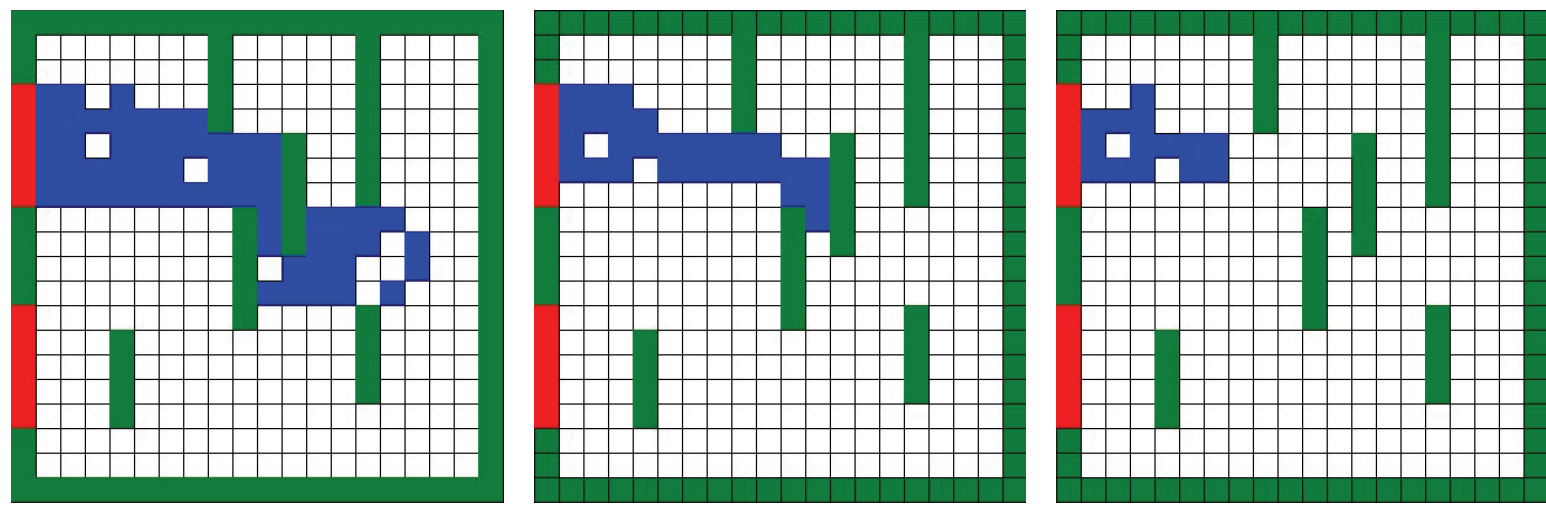

(a) Global-view model only
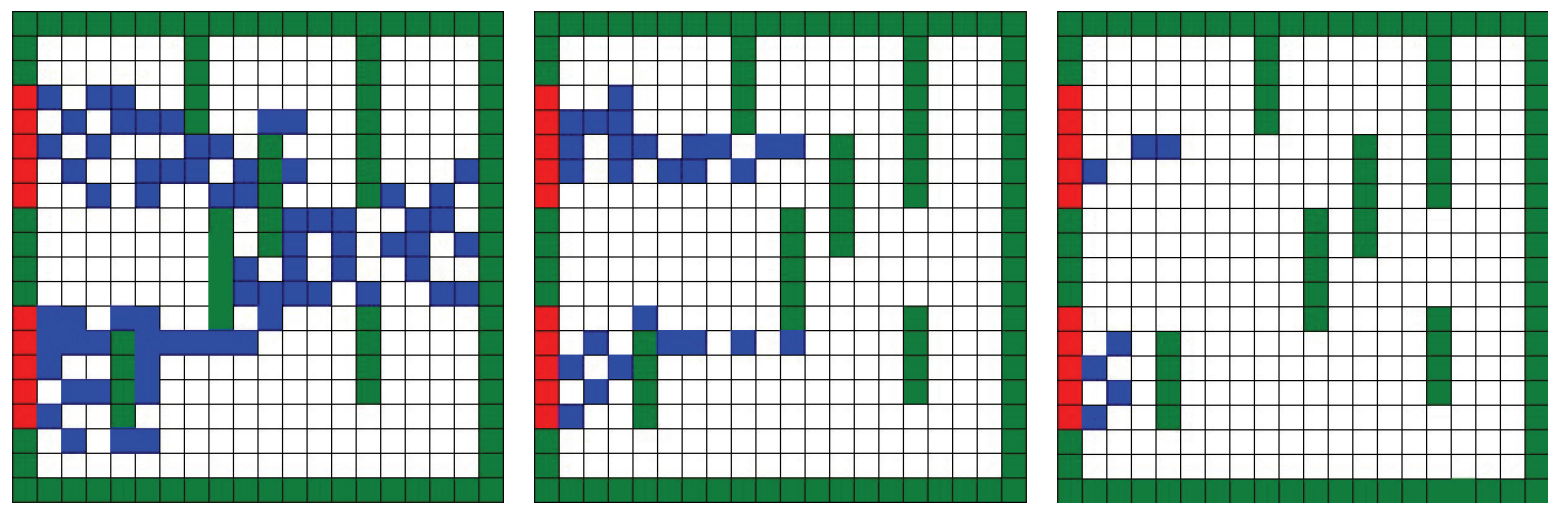

(b) Local-view model only
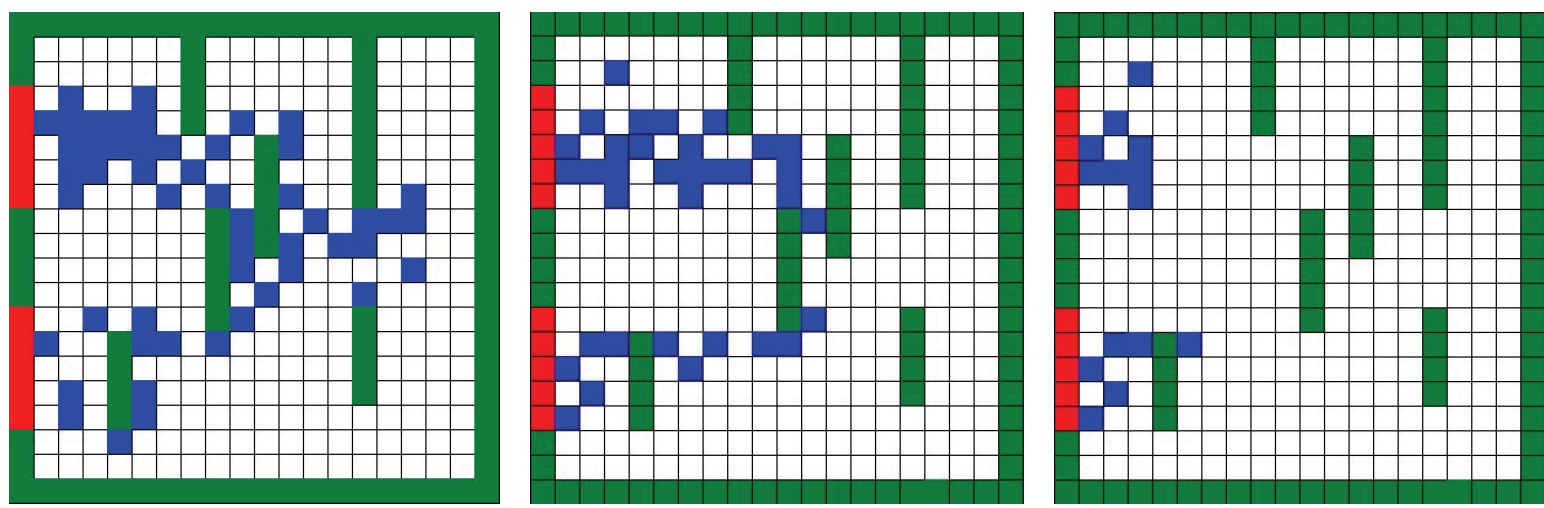

(c) Global-view and local-view

FIgURE 9: Pedestrian evacuation simulation in complex scenario.

the distance to the exit is less than the threshold; then use traditional methods of calculation.

We can see in Figure 4 that the dynamic floor field is more complex; it can be explained as the virtual path left by the pedestrian, and each step goes with the diffuse and decay to the neighbor cells; the diffuse probability is $\delta$, while the decay probability is $\alpha$, so the dynamic floor field can be regarded as a variable related to $\delta, \alpha$, and $t$.

3.1.3. The Local-View Floor Field and the Scenario-Familiarity. The local-view model assumed that the pedestrian can find the optimized goal in their visual range. Zeng et al. [8] proposed a local-view floor field model to solve the problem when pedestrians are unaware of the complex scenario (Figure 5); part of the pedestrians may feel blindness in the situation. The local-view floor field model performs better than the global-view model; it is common for a pedestrian to find a temporary goal as the destination, especially in some unfamiliar and complex environment, in people's real life. We analyze the local-view model proposed by Zeng et al. and join it with the standard global-view model; the result is much closer to the real situation.

As shown in Figure 5(a), when pedestrian is at the position A, they cannot see Goal A; although Goal A has 


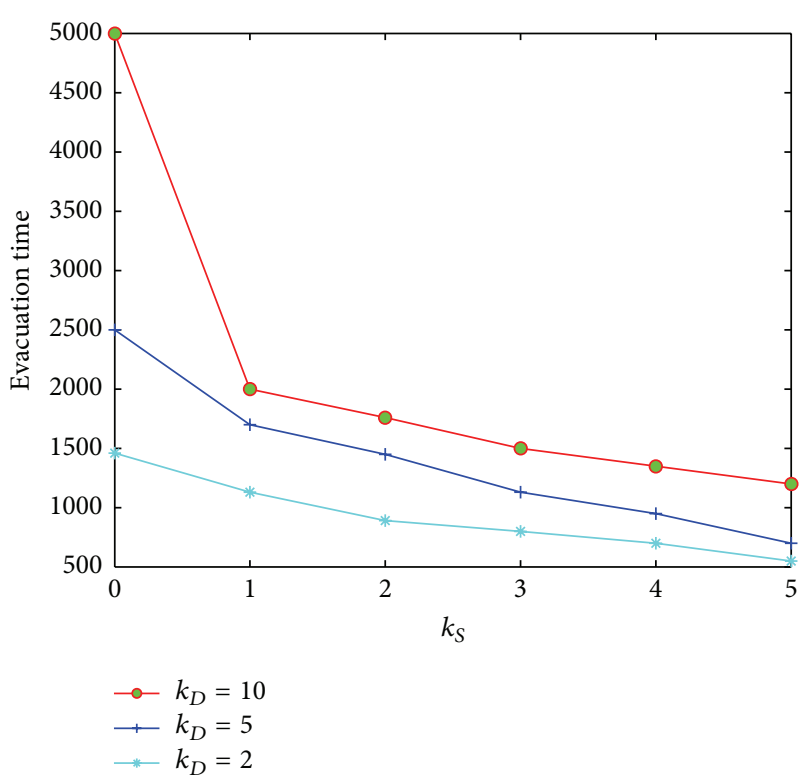

FIGURE 10: The relation between $k_{S}$ and the evacuation time.

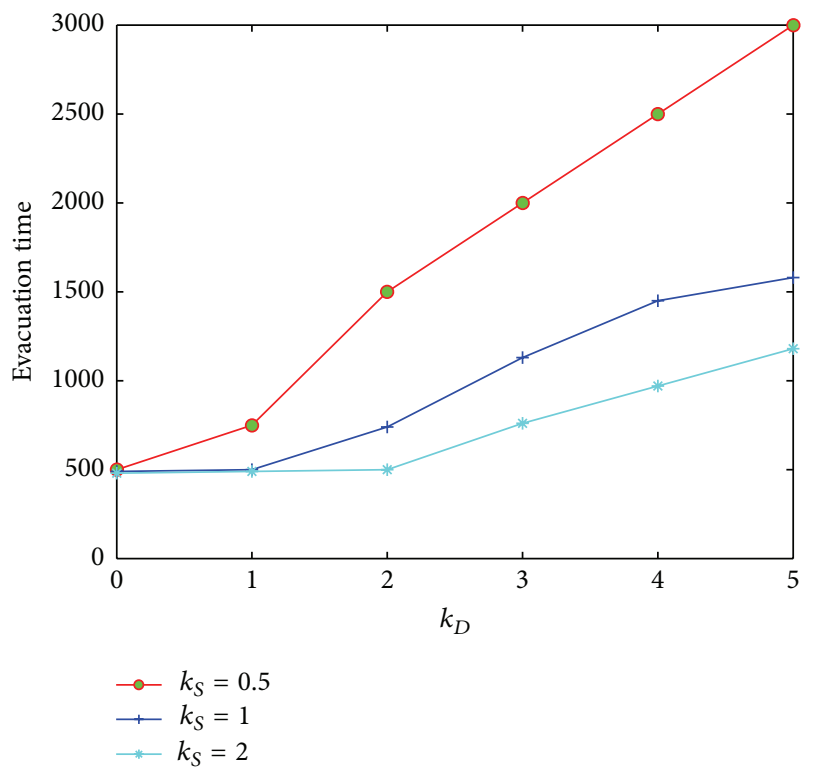

FIGURE 11: The relation between $k_{D}$ and the evacuation time.

an advantage over Goal B for them, they cannot see Goal B until they move to position B. In Figure 5(b), pedestrians at position $\mathrm{A}$ and position $\mathrm{B}$ have different goals because of the different visual range; the pedestrian at position $B$ cannot find the final destination, so he just finds a local-view destination instead, and the evacuation efficiency of local-view floor field model was validated to be better than the global-view model by Zeng et al. [8].

In our opinion, using single local-view model only is not live up to the matter of fact, because, in people's real life, part of people may be familiar with the complex scenario while the others are unfamiliar; also some people are in some ways familiar with the scenario. Aiming at this problem, we proposed the parameter of scenario-familiarity; $\lambda$ indicates the pedestrian familiar degree to the environment.

We analyze the evacuation efficiency of three models in complex scenario; we can see from Figures 6 and 7 that the local-view model has the best evacuation efficiency, because the pedestrian flow is dispersed. The pedestrians have known the final targets in Global-view model, so the pedestrian knows the way to get to the exit, and the density of the flow is high in this model. The global- and local-view model has the weaker efficiency compared to the local-view model only.

3.2. Update Rules. Update rules are mainly aimed at the pedestrian location in each time slice; the dynamic floor field is unified:

(a) The dynamic floor field is mainly influenced by diffuse and decay of neighbors.

(b) To every pedestrian, moving probability $p_{i j}$ is decided by static floor field and dynamic floor field:

$$
\begin{aligned}
p_{i j} & =\lambda N \exp \left(k_{D} D_{i j}\right) \exp \left(k_{S} S_{i j}\right)\left(1-n_{i j}\right) \zeta_{i j}, \\
N & =\left[\sum_{i, j)} \exp \left(k_{D} D_{i j}\right) \exp \left(k_{S} S_{i j}\right)\left(1-n_{i j}\right) \zeta_{i j}\right]^{-1} .
\end{aligned}
$$

(c) The target cell is chosen by the moving probability $p_{i j}$.

(d) If many pedestrians choose the same cell, compare their willingness probability.

(e) All the pedestrians use parallel update strategy.

\subsection{Experiment Flow}

(1) Initialization step contains some parameters initializations, such as pedestrian number $I$, static floor field weight $k_{S}$, and the dynamic floor field weight $k_{D}$ [13].

(2) After initialization, the target cell for pedestrian $i$ can be computed when $i<I+1$, and then check whether there are conflicts. Then work out all the conflicts. Part of the code is shown in Algorithm 1.

(3) Each pedestrian begins to move until they arrive at the exit when $t<T+1$. Part of the code is shown in Algorithm 2.

\section{Experiment Results}

We show the classic floor field model, pedestrian density $\rho=$ $0.7, k_{S}=2$, and $k_{D}=2$; the pedestrian evacuation in simple scenario is simulated; the process is shown in Figure 8; the $20 \times 20$ scenario is too simple, so we use $100 \times 100$ scenario instead to estimate the evacuation efficiency; the results of different models in complex scenario are shown in Figure 9.

4.1. Parameters. Figures 10 and 11 showed the change of the evacuation time when we fixed $k_{S}$ or $k_{D}$; it can be seen that the model we choose is similar to the Ansgar Kirchner model, so 

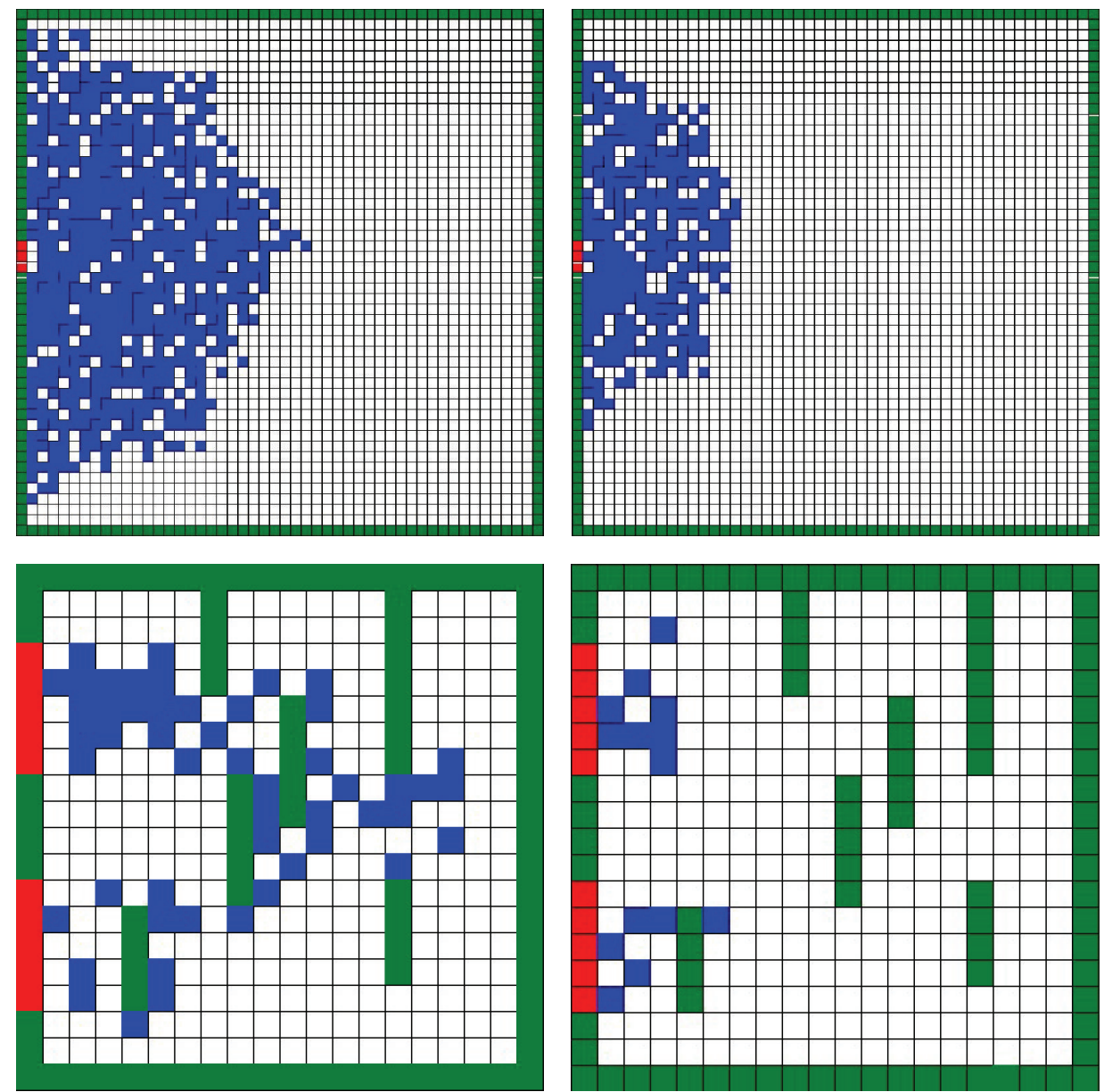

Standard

Use inertia

FIGURE 12: Inertia static floor field experiment.

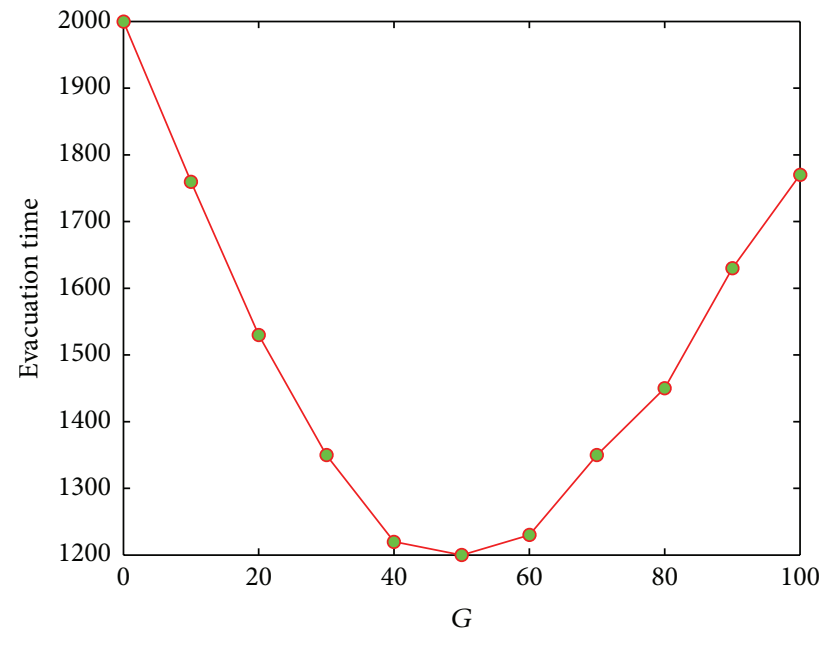

$\multimap$ Inertia SFF

FIGURE 13: Threshold and the evacuation time.

our experiment model is credible. The solid lines in Figure 10 are corresponding to $k_{D}=10, k_{D}=5$, and $k_{D}=0$. The solid
TABLE 2: Inertia static floor field.

\begin{tabular}{lcc}
\hline Evacuation time (MS) & Standard & Use inertia \\
\hline Simple scenario & 214200 & 164500 \\
Complex scenario & 375400 & 263200 \\
\hline
\end{tabular}

lines in Figure 11 are corresponding to $k_{S}=0.5, k_{S}=1$, and $k_{S}=2$.

4.2. The Inertia Static Floor Field. We mainly discussed the effect of the inertia static floor field and the value of $G$ on the evacuation time shown in Table 2; after 2000 steps, the evacuation result is shown in Figure 12; we can see the inertia static floor field is superior to the standard model.

The inertia static floor field threshold $G$ has strong relation with the indoor space and the number of the pedestrians, so we only analyze the relation between $G$ and total evacuation time in our model. The threshold $G$ can be explained when we bring static floor field into the algorithm. The relation between $G$ and the evacuation time is shown in Figure 13; we can find that there is an optimal value for $G$. 


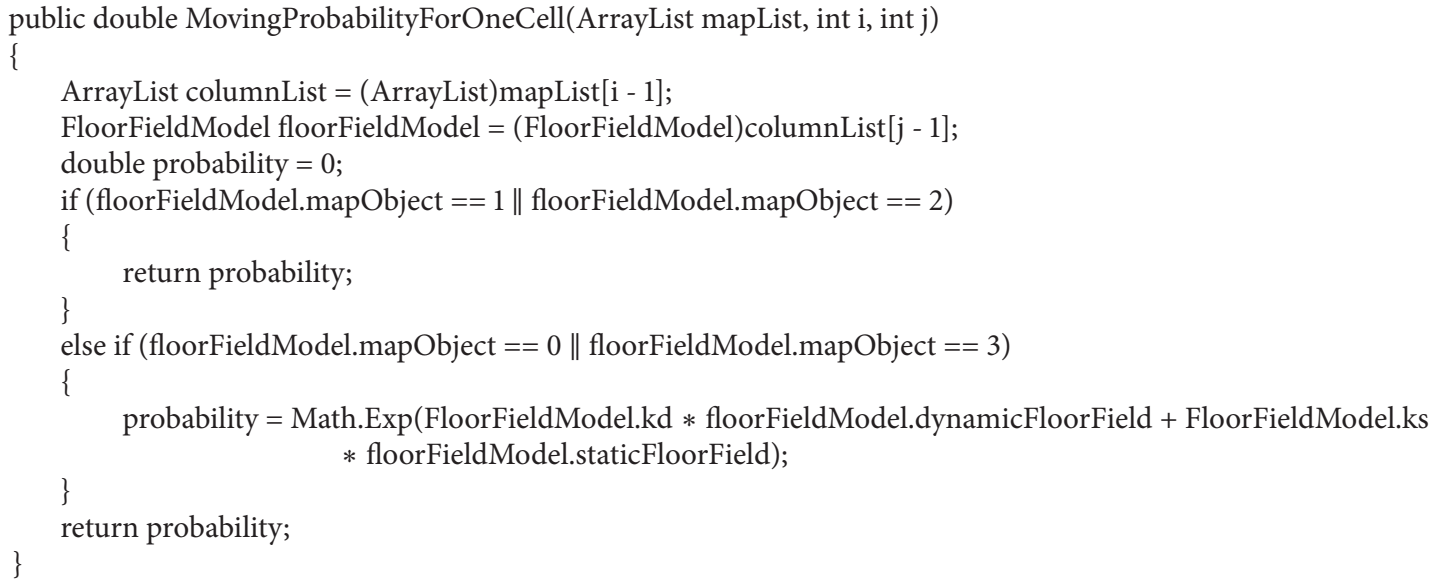

Algorithm 1: Part of the code.

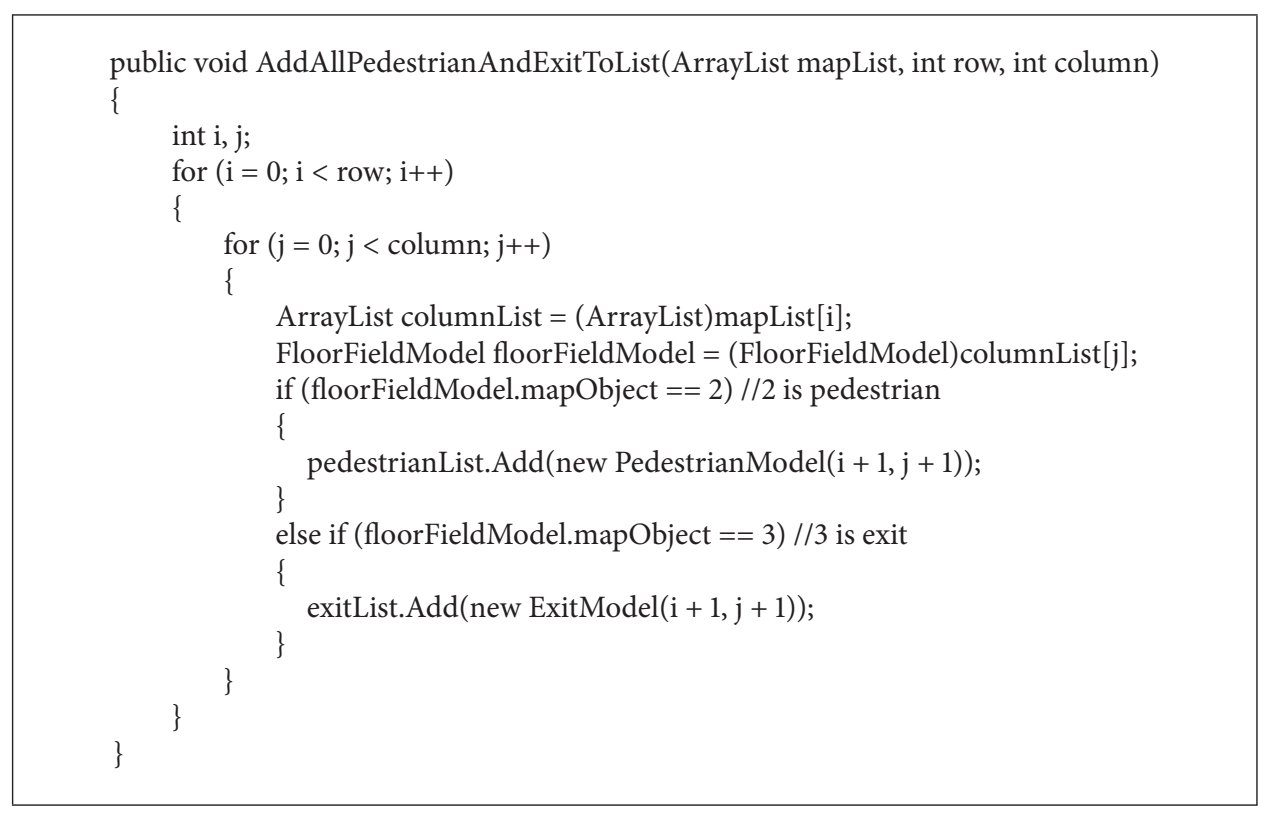

Algorithm 2: Part of the code.

\section{Conclusion}

In this paper, we established pedestrian flow simulation model with dynamic parameters based on cellular automata; the model assumes each pedestrian in the process of choosing the shortest possible route to the destination. Pedestrians during the move quickly and continuously optional on their own trade-offs in different positions, choose a more reasonable position as their next move target position. Model uses the weighting static and dynamic floor field to compute the move probability, detailing the definition of the parameters and the calculation methods of evacuation simulation.

On the basis of the actual analysis experiments Ansgar Kirchner model, we proposed an improved floor field model based on cellular automata. The update rules of the cellular automaton model fully restored classic phenomenon of various evacuation models; through simulation, we analyze the influence of parameters such as threshold values to the model results, show the problems that the interior space crowded evacuation may produce, and measured the optimal threshold of inertia static floor field. The proposed model is more suitable to simulate large plaza and complex structures, as well as some of the fire and other emergency evacuation situations.

Due to the different speeds and destinations of pedestrian movement in different scenes, so as to make the simulation more realistic, we should also introduce a variety of pedestrian movement speed processing mechanisms and clogging mechanism in the future. For depth study on different speeds, different purposes are a new direction. Pedestrians moving direction and the moving distance can be unified into a simulation vector [14]. In addition, in different densities 
and pedestrians congested states, the individual behavior characteristic of a pedestrian are different; the various social forces [15] factors should be taken into account.

\section{Conflict of Interests}

The authors declare that there is no conflict of interests regarding the publication of this paper.

\section{References}

[1] C. Burstedde, K. Klauck, A. Schadschneider, and J. Zittartz, "Simulation of pedestrian dynamics using a two-dimensional cellular automaton," Physica A: Statistical Mechanics and its Applications, vol. 295, no. 3-4, pp. 507-525, 2001.

[2] S. Wolfram, "Statistical mechanics of cellular automata," Reviews of Modern Physics, vol. 55, no. 3, pp. 601-644, 1983.

[3] A. Kirchner, H. Klupfel, and K. Nishinari, "Discretization effects and the influence of walking speed in cellular automata models for pedestrian 25 dynamics," Journal of Statistical Mechanics: Theory and Experiment, vol. 10, p. 10011, 2004.

[4] J. Ma, W.-G. Song, and G.-X. Liao, "Multi-grid simulation of pedestrian counter flow with topological interaction," Chinese Physics B, vol. 19, no. 12, Article ID 128901, 2010.

[5] R.-Y. Guo and H.-J. Huang, "A modified floor field cellular automata model for pedestrian evacuation simulation," Journal of Physics A: Mathematical and Theoretical, vol. 41, no. 38, Article ID 385104, 2008.

[6] D. Yanagisawa, A. Kimura, A. Tomoeda et al., "Introduction of frictional and turning function for pedestrian outflow with an obstacle," Physical Review E, vol. 80, no. 3, Article ID 036110, 2009.

[7] M. Chraibi, U. Kemloh, A. Schadschneider, and A. Seyfried, "Force-based models of pedestrian dynamics," Networks and Heterogeneous Media, vol. 6, no. 3, pp. 425-442, 2011.

[8] J.-B. Zeng, B. Leng, Z. Xiong, and Z. Qin, "Pedestrian dynamics in a two-dimensional complex scenario using a local view floor field model," International Journal of Modern Physics C, vol. 22, no. 8, pp. 775-803, 2011.

[9] A. Kirchner and A. Schadschneider, "Simulation of evacuation processes using a bionics-inspired cellular automaton model for pedestrian dynamics," Physica A, vol. 312, no. 1-2, pp. 260-276, 2002.

[10] A. Seyfried, M. Boltes, J. Kähler, W. Klingsch, and A. Portz, "Enhanced empirical data for the fundamental diagram and the flow through bottlenecks," in Pedestrian and Evacuation Dynamics 2008, pp. 145-156, Springer, 2010.

[11] Y. Jiang, H. Zhang, Z. Li et al., "Design and optimization of multiclocked embedded systems using formal techniques," IEEE Transactions on Industrial Electronics, vol. 62, no. 2, pp. 1270-1278, 2015.

[12] C. Saloma, G. J. Perez, G. Tapang, M. Lim, and C. PalmesSaloma, "Self-organized queuing and scale-free behavior in real escape panic," Proceedings of the National Academy of Sciences of the United States of America, vol. 100, no. 21, pp. 11947-11952, 2003.

[13] Y. Hao, Study on the SimulationModel of Pedestrian Flow Based on Cellular Automata, Beijing Jiaotong University, Beijing, China.
[14] J. Yang, Z. Hou, and M. Zhan, "Simulation of pedestrian dynamic using a vector floor field model," International Journal of Modern Physics C, vol. 24, no. 4, Article ID 1350023, 2013.

[15] D. Helbing and P. Molnár, "Social force model for pedestrian dynamics," Physical Review E, vol. 51, no. 5, pp. 4282-4286, 1995. 


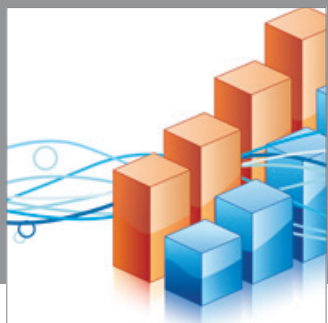

Advances in

Operations Research

mansans

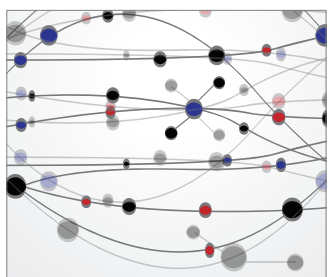

The Scientific World Journal
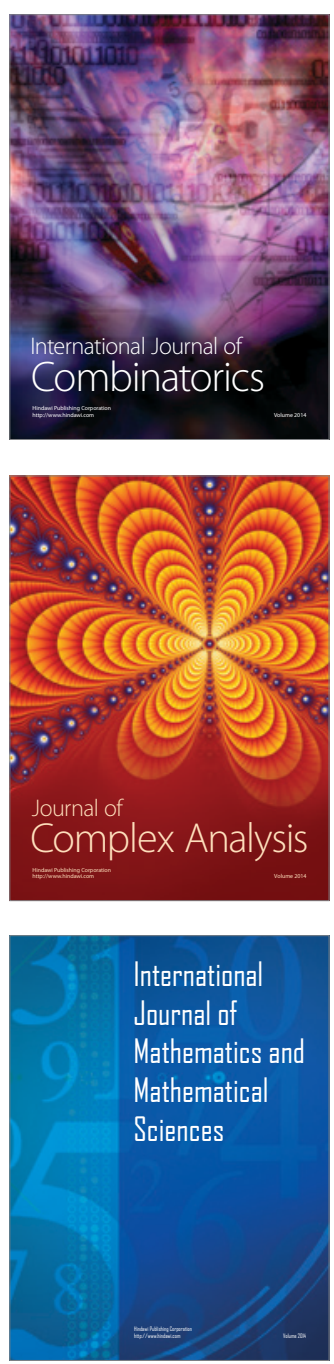
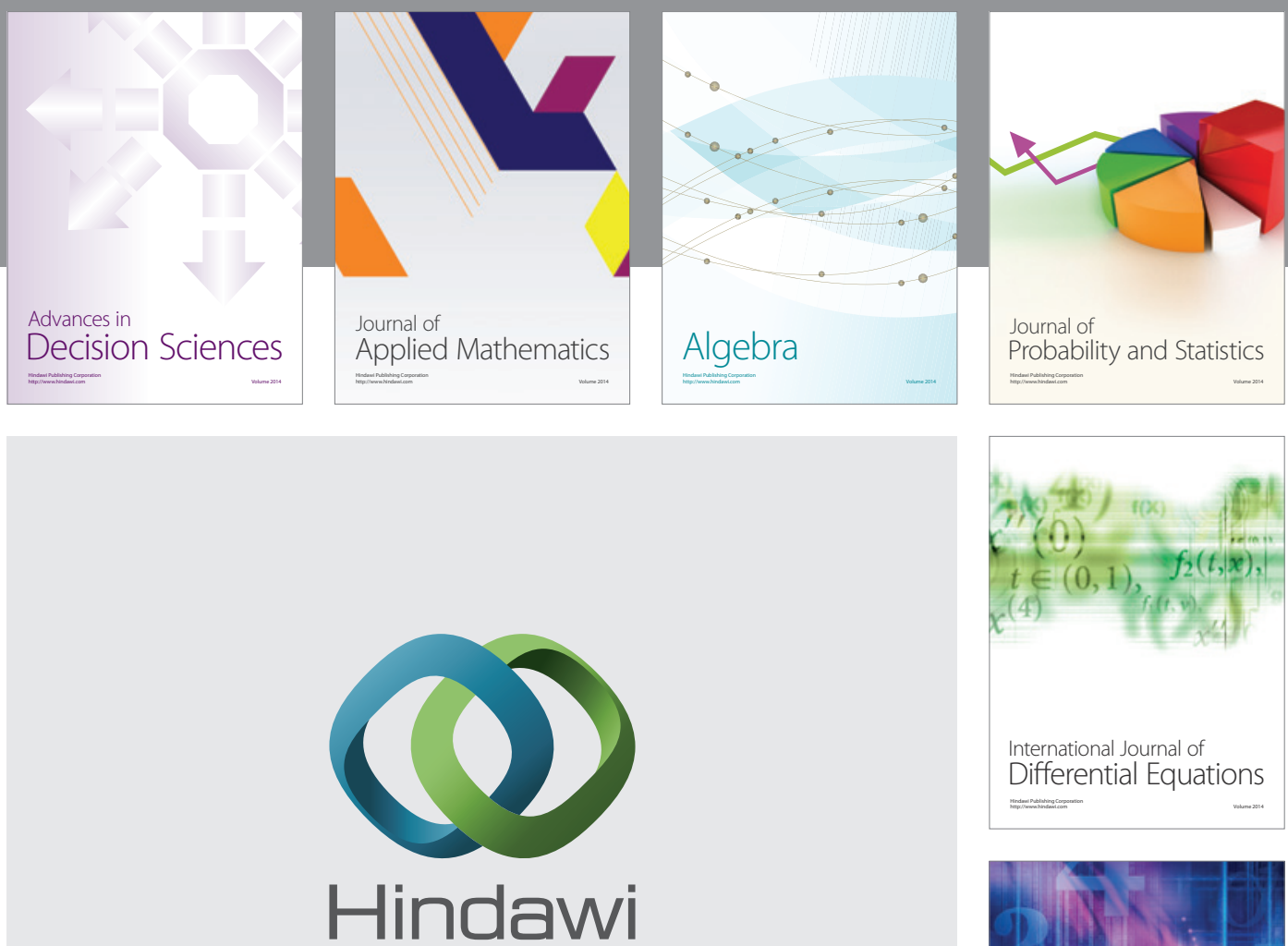

Submit your manuscripts at http://www.hindawi.com
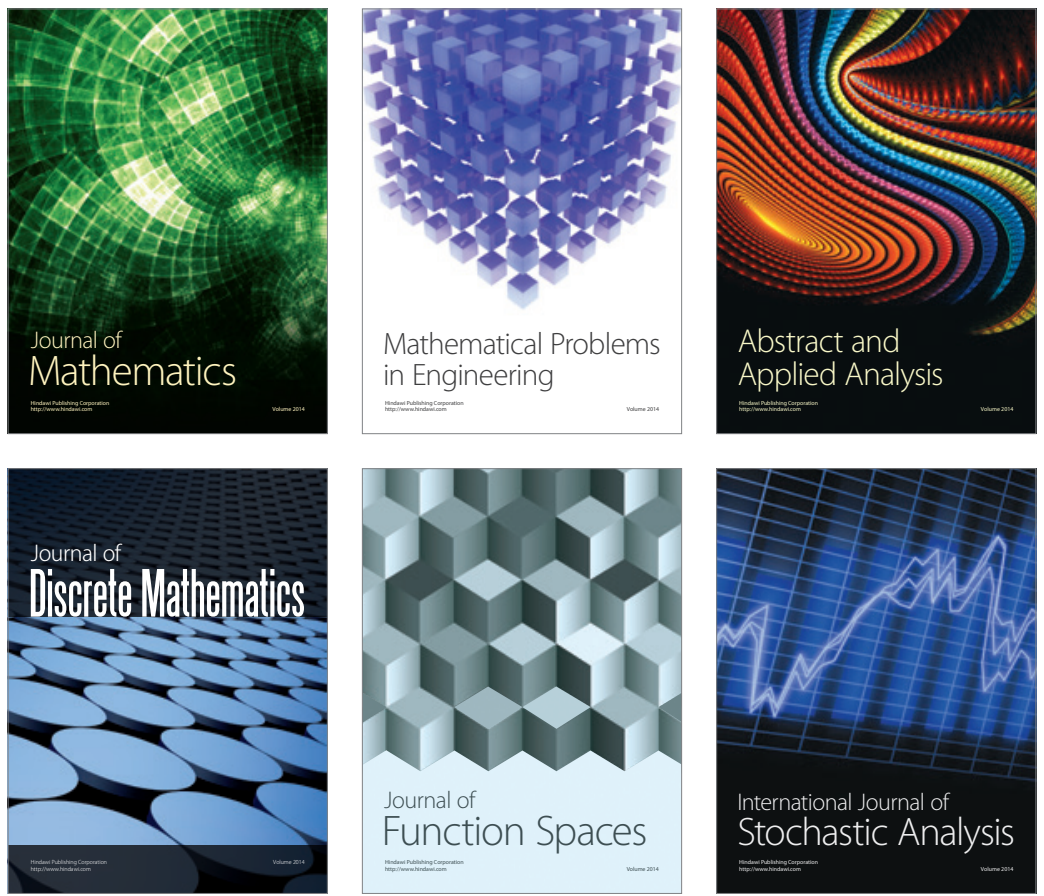

Journal of

Function Spaces

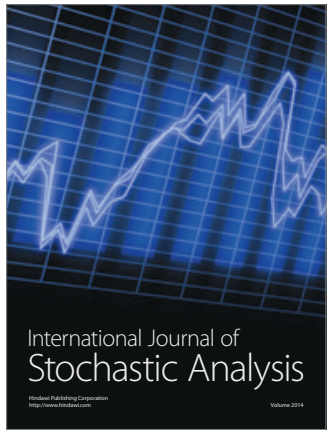

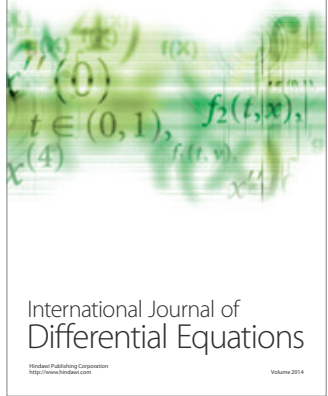
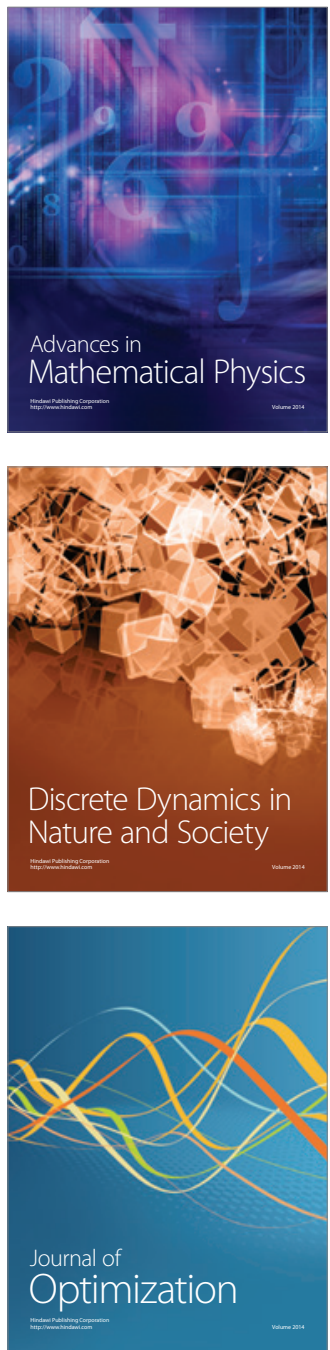